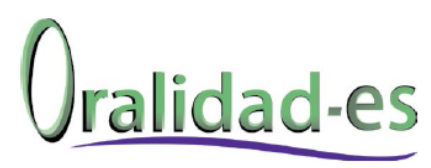

\title{
Tendencias temáticas e investigativas de la Red Iberoamericana de Estudios sobre Oralidad y sus aportes a la formación docente ${ }^{1}$
}

\author{
Thematic and investigative trends of the Ibero-American \\ network of studies on orality and their contributions to \\ teacher training.
}

\author{
Marleny Hernández-Rincón \\ marhdezrincon@gmail.com \\ Secretaria de Educación Bogotá, Colombia \\ Mirta Yolima Gutiérrez-Ríos \\ yolimagr@yahoo.es; mygutierrez@unisalle.edu. \\ co \\ Universidad de La Salle, Colombia
}

Hernández-Rincón, M. \& Gutiérrez-Ríos, Y. (2019). Tendencias temáticas e investigativas de la Red Iberoamericana de Estudios sobre Oralidad y sus aportes a la formación docente. Oralidad-es, 5, 1-14. https://revistaoralidad-es.com/index. $\mathrm{php/ro-es/article/view/112/82}$

Fecha de recepción: 18 de marzo de 2019 / Fecha de aprobación: 24 de junio de 2019 


\section{Resumen}

El propósito de este artículo es presentar las tendencias temáticas e investigativas de la oralidad en Iberoamérica. Se realizó una revisión bibliográfica al acervo de experiencias pedagógicas e investigativas presentadas en los congresos de la Red iberoamericana de estudios sobre la oralidad. Se analizaron 153 ponencias publicadas en las memorias de siete congresos realizados en Colombia, España y México entre 2011 y 2018, mediante el uso de una herramienta digital de análisis cualitativo basada en la hoja de cálculo. De este modo, se establecieron dos tendencias: la primera, relacionada con la oralidad como campo de estudio y la segunda, referida a la oralidad académica como eje de desarrollo educativo y profesional. Se reconoce la relevancia dada a los estudios sobre la oralidad y la consolidación de una comunidad de práctica académica e investigativa conformada profesores y académicos de diversos contextos escolares y sociales con aperturas creativas, críticas y de resistencia.

Palabras clave

Oralidad; experiencias investigativas; experiencias pedagógicas; red académica

\section{Abstract}

In this paper, the topic and investigative trends of orality in Iberoamérica are presented. It was made a literature review of the compendium of pedagogical and research experiences presented at congress of the Red iberoamericana de estudios sobre la oralidad. One hundred and fifty three papers published from the memoirs of seven conferences which took place in Colombia, Spain and Mexico between 2011 and 2018 were analyzed, by using digital qualitative analysis tools based on the spreadsheet. Thus, two trends were established: firstly, the one related to orality as a field of study and secondly, the one referred to academic orality as an axis of educational and professional development. It is recognized that studies about orality and consolidation of research and academic community practice formed by professors and diverse school and social contexts with creative, critical and resistance openings are relevant.

Key words:

Orality; research experiences; pedagogical experiences; academic network

\footnotetext{
${ }^{1}$ Este texto responde a los resultados iniciales de un estado de arte moderado sobre el desarrollo investigativo y pedagógico de la oralidad, en el marco de la tesis doctoral relacionada con el género discursivo oral desde la acción didáctica colectiva sistemática de maestros en el Grado Transición de la Educación Preescolar, dirigida por la Dra. Mirta Yolima Gutiérrez Ríos.
} 


\section{La oralidad en el escenario de una red académica}

En Iberoamérica, durante las últimas décadas se ha ido consolidando una comunidad de práctica académica e investigativa conformada por grupos de investigadores, en su mayoría docentes, interesados en estudiar la oralidad en diversos contextos escolares y sociales con el fin de comprender su naturaleza y los elementos teóricos y prácticos que la constituyen; es decir, sus perspectivas teóricas y las formas como se manifiesta en distintos escenarios de desarrollo humano y social.

\section{En el ámbito académico, en particular, tres} grupos de investigación de Colombia ${ }^{2}$ España $^{3}$ y México ${ }^{4}$ convocaron a otros investigadores de diferentes campos disciplinares a constituir y fortalecer la Red iberoamericana de estudios sobre la oralidad en el marco de congresos académicos bianuales que se vienen realizando desde el 2011 con el propósito de intercambiar saberes y experiencias en torno a la oralidad en diversos contextos. Este movimiento de investigadores ha ido organizándose en nodos-países y nodos regionales y en la actualidad a los tres ya mencionados, se han sumado, Chile, Argentina, Ecuador, Perú y Costa Rica. Estos esfuerzos, han contribuido a otorgar un estatuto a la oralidad como campo de estudio interdisciplinar con múltiples aristas en su comprensión y desarrollo.

Por otra parte, en Colombia se realizan dos estudios, uno relacionado con las perspectivas teóricas, tensiones y aperturas de la oralidad en Iberoamérica (2000 -2012) y otro, alrededor de las concepciones y prácticas de la oralidad en Colombia (Gutiérrez-Ríos, 2011; 2014); en estos estudios se identificaron cuatro perspectivas epistémico-teóricas de la oralidad: 1) la perspectiva antropológica y socio cultural, 2) la literaria, 3) la retórica, lingüistica y discursiva, y 4) la perspectiva educativa, pedagógica y didáctica. Estas han servido de horizontes temáticos para la organización y discusión en los congresos de la Red de oralidad.

Las experiencias pedagógicas e investigaciones y en general, las contribuciones presentadas en los congresos de la Red de Oralidad, fueron compiladas en memorias, libros y actas de los congresos (Pinilla y Gutiérrez, 2012; Núñez, Rienda \& Santamarina, 2013; Rodríguez \& Pinilla, 2014 y Quitián \& Gutiérrez-Ríos, 2016). Y hoy constituyen un acervo importante para abordar la oralidad como objeto de estudio.

Dados los anteriores aportes, surge el interés por actualizar los desarrollos investigativos y pedagógicos de la Red iberoamericana de estudios sobre la oralidad, en la perspectiva de desentrañar nuevos avances, tensiones y vacíos de conocimiento con respecto a la oralidad como objeto de enseñanza y aprendizaje. Para tal fin, se realiza un estado de arte moderado, a partir de las investigaciones y experiencias desarrolladas entre 2011 y 2018, con el objetivo de indagar por: ¿Cuáles son las tendencias temáticas e investigativas de la Red iberoamericana de estudios sobre la oralidad en la última década?

Se realizó una revisión de las memorias publicadas en 7 congresos organizados por la Red de oralidad (5 internacionales y 2 nacionales), la cual dio como resultado un corpus constituido por 153 ponencias o comunicaciones, entre las que se destacan resultados de investigación y experiencias pedagógicas relacionadas con las perspectivas de la oralidad mencionadas.

\footnotetext{
${ }^{2}$ El grupo Lenguaje, Cultura e Identidad de la Universidad Distrital, realiza el Primer encuentro iberoamericano de estudios sobre oralidad. La oralidad en contextos diversos (en Bogotá el 8, 9 y 10 de junio del 2011)

${ }^{3}$ El grupo de investigación Étimo de la Universidad de Granada, realiza el Segundo encuentro Iberoamericano de estudios sobre oralidad y V Encuentro Latinoamericano de Identidades, realizado durante los días 9,10 y 11 de octubre en la Universidad de Granada (España)

${ }^{4}$ El grupo Oralidad, Tradición y Cultura Popular y la Facultad de Ciencias Sociales, Universidad Nacional Autónoma de México

(UNAM), realizan el Tercer encuentro iberoamericano de estudios sobre oralidad (en México D.F. 5, 6 y 7 de octubre de 2015
} 
Tabla 1.

Categorias de análisis y contraste.

\begin{tabular}{|l|l|}
\hline Nombre & Características \\
\hline Contexto académico & Congreso en el cual se presentó la ponencia \\
\hline Población & Universo o muestra de estudio \\
\hline Nivel educativo & Ciclo o grado escolar de la población \\
\hline Problema & $\begin{array}{l}\text { Necesidades que originan la investigación o la } \\
\text { experiencia pedagógica }\end{array}$ \\
\hline Tema & Asunto que estudia \\
\hline Objetivo & Fin o meta a alcanzar \\
\hline Fundamentos & Perspectiva y concepto de la oralidad \\
\hline Metodología & Diseño metodológico \\
\hline Conclusiones & Hallazgos o resignificaciones \\
\hline Prospectiva & Proyectos y desafíos \\
\hline
\end{tabular}

Fuente: elaboración propia

El análisis del corpus se realizó con base en la técnica matricial para el análisis cualitativo de datos que consistió en la triangulación de la información mediante matrices elaboradas en formato Excel (hoja de cálculo). Esta herramienta digital facilita la organización de la información recolectada y la yuxtaponen desde diferentes ángulos, favoreciendo su triangulación (Kalman \& Rendón, 2016) mediante el establecimiento de relaciones por temas, problemas y datos específicos del contexto. Por tanto, se organizaron las categorías de análisis codificadas en una tabla dinámica que permitió sistematizar la información, visualizarla de diferentes maneras y

Figura 1.

Ejes temáticos de la oralidad.

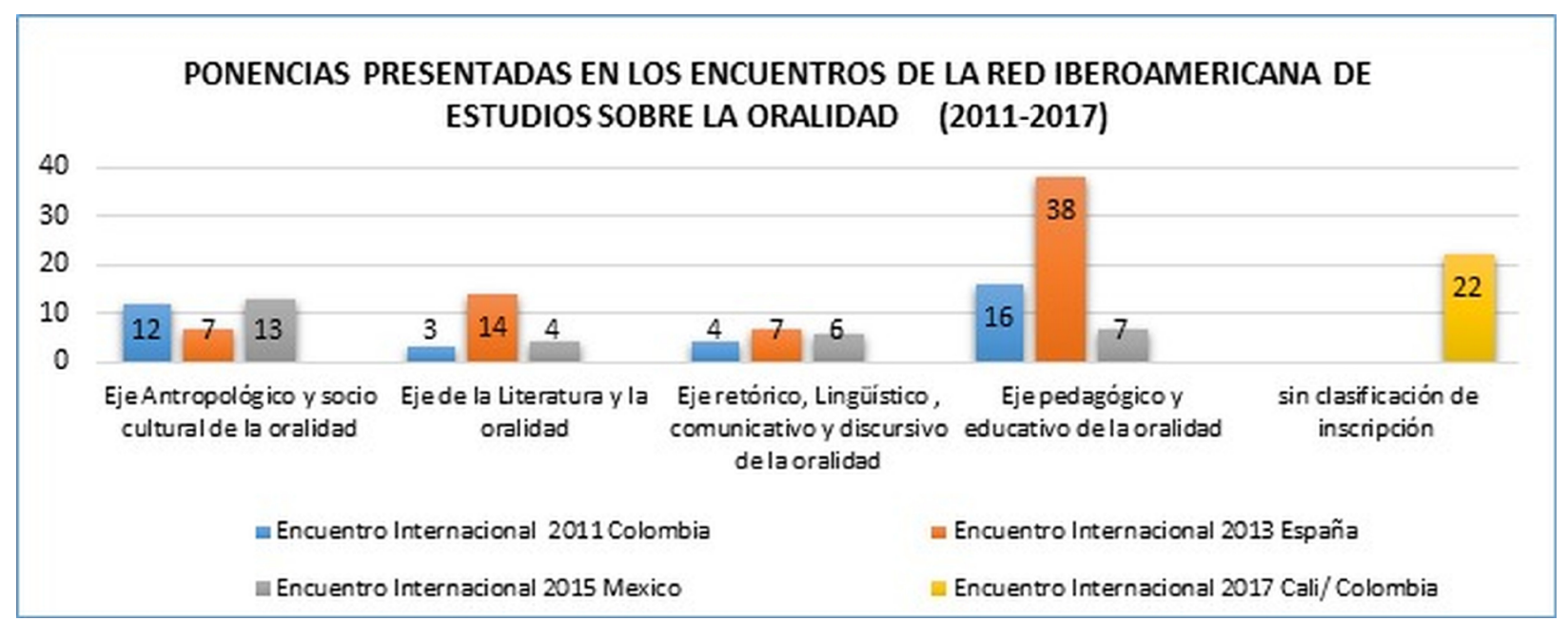

Fuente: Memorias eventos académicos realizados entre 2011 y 2018. 
descubrir nuevas relaciones o encontrar nuevas categorías. La Tabla 1 muestra las categorías de análisis y contraste.

Una vez se diseñó la hoja cálculo según las necesidades investigativas, se realizó un primer filtro de análisis que permitió confirmar los ejes temáticos de la oralidad que habían sido hallados en una revisión preliminar (Gutiérrez-Ríos, 2014) e identificar un conjunto de temas emergentes $y$, por tanto, nuevos ejes temáticos, como se ilustra en la Figura 1.

De manera similar se establecieron diferentes contrastes de información según las categorías o filtros de la hoja cálculo que permitió una lectura detallada de los datos registrados y de este modo, se establecieron dos tendencias temáticas e investigativas de la Red de oralidad: La primera, relacionada con la oralidad como campo de estudio y la segunda tendencia, referida a la oralidad académica como eje de desarrollo educativo $y$ profesional.

\section{Tendencia 1. La oralidad como campo de estudio}

Dado que, uno de los principales propósitos de la Red iberoamericana de estudios sobre la oralidad, desde su constitución (2011), ha sido reunir un acervo de investigaciones y experiencias pedagógicas relacionadas con la oralidad en diversos contextos, se confirma la existencia de la oralidad como un campo de estudio con una serie de problemas específicos presentes en diferentes disciplinas de las Ciencias sociales y las humanidades. Así mismo, se identifican distintas manifestaciones de la oralidad en contextos escolares y sociales, las perspectivas teóricas y metodológicas que le subyacen; la emergencia de nuevas problemáticas y, por tanto, de futuras líneas de investigación.
En el corpus analizado se evidencia un abanico de significados y sentidos dados a la oralidad, según los intereses y necesidades investigativas y pedagógicas de los autores. Por ejemplo, en las ponencias y comunicaciones presentadas en el eje antropológico y socio cultural, predomina la concepción de oralidad como instrumento de supervivencia y desarrollo de la vida social. La oralidad es el elemento que funciona en un presente con cierta estructura predeterminada a partir de la acción de transmisión de la palabra (Agudelo \& Sanabria, 2012; Delgadillo, 2014; Obando, 2014) y funciona no solo como signo lingüístico, sino también en torno a las particularidades de la expresión fonética, ritmos, tonos, énfasis que se utilizan en elementos heredados de las culturas (Hamui, 2012).

Además, las concepciones, características y condiciones del diálogo en familias rurales colombianas dan cuenta de la singularidad de temas, espacios, tiempos, personas con quien se dialoga, condiciones, repercusiones en la vida cotidiana y en la paz, lo que permite visualizar tradiciones y prácticas en la resolución de conflictos (Páez Martínez, Del Valle Idárraga, Gutiérrez-Ríos \& Ramírez-Orozco, 2016; Beltrán \& Arango, 2012).

Este eje antropológico y socio cultural de la oralidad recoge desde los albores del lenguaje oral el devenir de la humanidad, evidente en las palabras cargadas de simbolismo religioso y científico (Peña, 2012; Méndez, 2012). Por tanto, reconocer la naturaleza sociocultural de la oralidad, implica remontarse a las sociedades primigenias, para identificar su funcionamiento materializado en sonidos y movimientos corpóreos y cómo en la interacción construye, expresa y comunica sentidos (Contreras, 2014).

En las contribuciones realizadas en el eje educativo, pedagógico y didáctico de la oralidad, diferentes investigadores brindan elementos para fundamentar los usos dados a la oralidad y a sus dinámicas como constructoras de realidades; 
así como distintas estrategias para ingresar a la vida social y construir condiciones de ciudadanía propias de una ética de la comunicación oral (Carvajal, 2012; Rodríguez, 2012; Camelo, Cárdenas, Torres, Gutiérrez-Ríos, Rodríguez \& Pinilla, 2011). Es decir que el papel de la oralidad en la constitución de sujeto y en los procesos de mediación e interacción, ha ido configurando un importante campo de análisis (Obando,2014; Núñez, 2012; Gutiérrez, 2012) y plantea la discusión sobre la necesidad de su estudio en el campo de la didáctica de la lengua oral en diferentes los diferentes niveles de educación (Carvajal \& Quitián 2014).

Por otra parte, se problematiza la ausencia de criterios de evaluación en la enseñanza y aprendizaje de la oralidad. Por ejemplo, Vásquez (2012), plantea que la enseñanza de la oralidad implica la incorporación de una gran variedad de conectores o bisagras discursivas que permiten superar el habla fragmentada sin ninguna lógica. Es decir que el poner a circular la oralidad y sus modalidades en los espacios educativos, "no sólo busca favorecer la interacción entre los estudiantes sino, que educa en la convivencia, la inclusión y la construcción de la democracia” (p.358).

Razón que permite establecer varios campos investigativos para abordar la oralidad desde una perspectiva evaluativa. Sin embargo, no hay una postura crítica para asumir la oralidad desde este componente. Al respecto Hernández \& Chávez (2016) postulan un acercamiento desde la evaluación dialógica como estrategia para promover la interacción en el aula y constituyen el portafolio oral como una forma de hacer evidente las destrezas orales en el contexto escolar.

En el eje retórico, lingüístico, comunicativo y discursivo de la oralidad, se considera la palabra oral como expresión en un espacio compartido y solidario. Se evidencia una tendencia de mirar en detalle los actos enunciativos y dominios de la actividad comunicativa que da complejidad a la estructura del lenguaje y la comunicación. Con- fluye la concepción de una oralidad que acontece en el deliberado uso de la palabra que constituye sentidos y es una práctica social y cultural de naturaleza dialógica y multimodal (Gutiérrez-Ríos, 2012, 2018 )

Del mismo modo, el eje literatura y oralidad sigue constituyendo su perspectiva desde las posibilidades de reflexionar sobre el lenguaje y su relación con la acción poética como formas de simbolización, comprensión, interpretación y expresión del mundo. Esta condición destaca el aporte de las investigaciones por preservar las lenguas indígenas, valorar sus tradiciones orales al traducirlas y transcribirlas (Sanagogo \& Yopasa, 2012).

Por esto, las culturas orales conservan sus conocimientos por medio de narraciones que representan una poesía narrativa que incorpora el arte de la palabra, por tal motivo se reconoce la literatura oral, como literatura tradicional en sus múltiples formas, que puede convertirse en recurso didáctico para otras áreas de conocimiento, al margen de la lengua y la literatura (Rienda, 2012). Por todo esto se fundamenta el carácter crucial de la literatura y reencontrar el equilibrio entre la literatura como fruición y su vertiente educativa. En general, este rastreo de información logra establecer la tendencia de la oralidad, como el eje de fundamentación teórica y la ventana de oportunidades a las que puede tener acceso la escuela al pensarse en una pedagogía de la oralidad como parte esencial del saber pedagógico, que quebranta la mirada de lo espontáneo y natural en el habla. De esta manera se configura la oralidad como un campo de estudio con un valor epistémico, metodológico y unos principios propios, que determinan la aceptabilidad de la producción oral, su naturaleza e intencionalidades en el contexto de enseñanza y aprendizaje (Gutiérrez-Ríos, 2018).

Desde esta tendencia de la oralidad como campo de estudio se invita a pensar la construcción de la voz de los sujetos en el marco de interacciones 
del yo con el otro, cifrado en un nosotros en el plano de alteridad planteado por Bajtín (1982), lo cual influirá en la construcción de la identidad y en el reconocimiento del rol de agentes social vinculado a una comunidad (Zarate \& Fajardo, 2017). De esta manera, la oralidad se posiciona como campo de estudio articulada a distintas disciplinas desde su naturaleza de práctica social y cultural humana.

\section{Tendencia 2: La oralidad académica como eje de desarrollo educativo $y$ profesional}

Los avances identificados con respecto a la oralidad muestran una tendencia al desarrollo educativo y profesional en contextos escolarizados (preescolar, primaria, secundaria y superior) y en contextos de acción laboral y profesional. Desde esta postura se justifica la presencia de la enseñanza y el aprendizaje de la oralidad formal o académica en los planes de estudio y la necesidad de que la escuela reconozca la relación entre lo prototípico (informalidad en la vida cotidiana) y lo periférico (estructura formal) para abordar la oralidad desde su uso en el contexto escolar.

Por tanto, esta tendencia respecto a la presencia de la oralidad académica en el ámbito escolar y social tiene una serie de complejidades que ameritan su estudio. En las investigaciones de Jurado (2014), Vásquez (2014), Amber \& Santamarina (2013) y Gil (2017), se encuentra un valioso análisis de la oralidad académica en los currículos y planes de estudio en países de habla hispana, con avances en la educación básica y media. Se reconoce, el andamiaje dado a la oralidad desde las experiencias docentes y particularmente, desde su carácter mediador y estratégico enfocado a fortalecer las interacciones verbales y por medio de estas, resignificar realidades y reconocer diferencias en la construcción de identidad individual y colectiva (Alfonso, Álvarez, Carreño, Martínez, Moreno, Nomelín, Saavedra y Tarquino, 2012).

Sin embargo, la oralidad sigue teniendo un lugar marginal con respecto a las prácticas de lectura y escritura académicas; si bien, en países como Argentina, Chile, México, Guatemala y Colombia $^{5}$ se evidencia que la enseñanza de la oralidad propicia el desarrollo de habilidades comunicativas, aún no se logra precisar sus alcances en la formación de sujetos discursivos en los diferentes niveles y contextos educativos. Además, se reitera la necesidad de estudiar la incidencia de la oralidad en y desde la práctica pedagógica dado su carácter dialógico. La perspectiva pedagógica de la oralidad es, entonces, "una práctica ética y, por consiguiente, también política. No existe práctica educativa sin intencionalidad; no existen posiciones neutrales en ella en relación con sujetos, concepciones, situaciones y contextos" (Ortega, 2009, p.31).

Por esta razón, se considera esta tendencia en desarrollo bajo los presupuestos de los mismos investigadores que en sus estudios plantean la necesidad de incorporar y actualizar conocimientos teóricos y prácticos acerca de cómo se desarrolla y trabaja la oralidad en el contexto educativo.

Al respecto, Núñez (2012) plantea la necesidad de fundamentar la oralidad en el campo de la educación y el compromiso del profesorado por una práctica investigadora y transformadora, para que la escuela reserve la oralidad como destreza lingüística enseñable y aprendible, que construye significados socialmente y fundamentan una didáctica en este campo, y se configura, como una invitación para continuar ganando terreno en la enseñanza de la oralidad formal en la escuela y se hace necesario seguir trabajando en la transformación de las concepciones disci-

\footnotetext{
${ }^{5}$ Primer estudio proyecto Iberlingua (OEI) y Segundo Estudio regional comparativo y explicativo de la calidad de la educación (Serce).
} 
plinares y didácticas de los docentes en todas las áreas de conocimiento (Gutiérrez- Ríos, 2012; Quitián, 2014).

En ese sentido, la oralidad en los contextos educativos de secundaria y formación universitaria tiene una gran demanda en relación con los usos de la oralidad para reproducir los aprendizajes, por lo cual se asume desde una perspectiva evaluativa de la lengua oral formal en el aula. Las investigaciones muestran el predominio de actividades relacionadas con el desarrollo de géneros orales como exposiciones, debates, mesas redondas y relatos (Camelo y otros, 2012). Se advierte, la necesidad de desarrollar estrategias didácticas que potencien el discurso oral académico y la necesidad de generar mayor conciencia, tanto del estudiante, como del docente, en la importancia de abordar la oralidad académica situada y formar docentes en las facultades de educación y en las escuelas normales superiores con y desde prácticas dialógicas que fortalezcan el pensamiento crítico (Chaparro \& Camargo, 2012; Gutiérrez-Ríos, Varga, Vives, Lara \& Uribe, 2016).

En relación con el contexto educativo de básica primaria, la tendencia de la oralidad busca posicionarse desde las interacciones que comprenden aspectos de subjetividad e intersubjetividad (Pinilla, 2012, 2014) y reorienta las relaciones que se establecen por medio de las palabras, las cuales aluden a procesos de oralidad y metacognición (Aramburo, 2017) y busca sensibilizar a los estudiantes de estos grados en la construcción simbólica de los elementos lingüísticos y extralingüísticos, con el objetivo de explorar variedad de alternativas semióticas, en dónde confluyan las palabras, gestos y espacios que aparecen como instrumentos de comunicación

Respecto a la adquisición y desarrollo de la oralidad en la Educación Preescolar se resaltan aspectos que buscan incentivar los actos de habla vinculados a la producción de sentido (Martínez, Moreno \& Vergara, 2012). Por tanto, la apertu- ra de actividades en este contexto confluye en la construcción de significados en contextos de interacción oral mediante distintas estrategias didácticas como los proyectos que comprometen la acción de los participantes en los eventos comunicativos. La importancia de este contexto (Galeano, 2012; Albarracín, Hernández, Urrea \& Villamil, 2012) es que logra posicionar al maestro como líder en la formación integral de los estudiantes y de allí la importancia de reflexionar sobre sus prácticas pedagógicas, ya que dan un lugar importante a la oralidad respecto a su injerencia en la construcción de ciudadanía y reconoce al niño como locutor e interlocutor válido en los procesos comunicativos (Rincón, Patiño \& Gómez, 2017).

En este ciclo escolar la relación que se establece entre profesores y estudiantes se lleva a cabo a través de procesos interactivos que deben ser entendidos como procesos de comprensión e interpretación mutua. Dichos procesos están relacionados con aspectos del uso de la lengua que demarcan distintas formas de materializar el discurso oral. Las investigaciones que se ocupan de entender la complejidad del proceso se han centrado en aspectos funcionales, cognitivos o socioculturales; que buscan explicar como un infante se convierte rápidamente en hablante que puede comunicarse con las personas de su entorno (Shiro, 2012).

Desde esta perspectiva, la oralidad en la educación inicial brinda oportunidades para entender cómo se adaptan los enunciados al contexto y al interlocutor y, para ello, es necesario examinar los procesos vinculados a la identificación y producción temprana de géneros discursivos orales. Por todo lo anterior, la enseñanza y el aprendizaje de la oralidad se evidencia en experiencias pedagógicas donde el maestro reconoce la necesidad de planear, implementar, revisar, evaluar y potenciar la adquisición y desarrollo de la oralidad del estudiante y con ello, cualificar su práctica docente (Villanueva, 2012; Rincón, 2015; León, 2015). 
Los estudios también muestran que la escucha es una práctica esencial y constitutiva de la oralidad; su presencia es fundamental en géneros discursivos orales. Por ejemplo, se plantea la relación escucha-conversación, a fin de comprender la necesidad del reconocimiento de la diferencia y las singularidades de los sujetos (Rojas, 2017). Por ende, se reclama la importancia de formar en la escucha desde los diversos contextos con un compromiso ético y pedagógico (León, 2019).

Esta tendencia investigativa de la oralidad académica y en particular de la necesidad de formar en una ética de la escucha, que forme en la capacidad de interactuar con la tecno cultura y armonizar las subjetividades ansiosas, dispersas e impacientes en la dialogicidad, esa capacidad humana de concebir, crear, reflexionar y comunicar realidades sociales.

Para tal fin, se requiere la formación de docentes que posean "una buena capacidad para seleccionar con autonomía y objetividad aquellos contenidos/temáticas/ saberes que necesitan ser enseñados y, por supuesto, aquellos métodos/ temáticas/saberes/ metodologías para hacerlos enseñables" (Álzate, 2015, p10). Esto conlleva a "explorar-se, sensibilizar-se, y observar-se desde su quehacer cotidiano, moviliza finalmente a sus estudiantes-comunidad hacia el crecimiento intelectual” (Álzate, 2015, p.10).

\section{De las aperturas y tensiones encontradas}

El acierto de constituir una Red de estudios sobre la oralidad como una comunidad académica e investigativa posibilita el encuentro reflexivo y crítico de investigadores nacionales e internacionales con un interés común, la oralidad y sus múltiples formas de configuración. En ese escenario, los aportes de las distintas disciplinas de las Ciencias Sociales y Humanas le han dado el estatuto de oralidad como campo de estudio y eje de desarrollo educativo y profesional.

La tendencia de la oralidad académica en el ámbito de la educación brinda posibilidades para avanzar en el desarrollo de condiciones pedagógicas y didácticas en todos los niveles de escolaridad, por cuanto es evidente la preocupación del profesorado por mejorar sus prácticas de enseñanza de la oralidad desde distintas estrategias.

En este sentido, también se avanza en la formación docente desde las estrategias de negociación y mediación científica, social y cultural, lo que posibilita el diseño de currículos transformadores desde perspectivas críticas y reflexivas que consolidan fundamentos epistemológicos, culturales, antropológicos y pedagógicos en la educación.

Los análisis realizados permiten establecer metodologías que recogen elementos y relaciones pedagógicas y didácticas que demuestran que en el campo de la oralidad lo que se consideraba una verdad absoluta, hoy es concebida como una verdad relativa, ya que las investigaciones presentadas por los maestros en los congresos dejar entrever un profesional autónomo, reflexivo, crítico e investigador de su propia práctica.

La oralidad y sus aportes en la formación docente inducen en las mediaciones que se realizan sobre la acción pedagógica. En otros términos, se hacen visibles elementos de una interacción con una visión social, de compromiso y de apertura. 


\section{Referencias}

Agudelo, E., \& Sanabria, M. (2012). Tapi, Juti y Wapa: Relación entre palabra y cultura material en diseños tradicionales Sikuani del Resguardo Wacoyo. En R. Pinilla, \& Y. Gutiérrez-Ríos, La oralidad en contextos diversos. aportes investigativos para su discusión y comprensión (págs. 61-72). Bogotá: (UD) Universidad Distrital Francisco José de Caldas.

Albarracin, R., Hernández, M., Urrea, D., \& Villamil, D. (2012). Pintando un cuadro de voces: Un análisis sobre los modos de habla del maestro en el aula de clase. En R. Pinilla, \& Y. Gutiérrez, La Oralidad en contextos diversos. Aportes investigativos para su discusión y comprensión (págs. 461-472). Bogotá: (UD) Universidad Distrital Francisco José de Caldas.

Alfonso, A., Carreño, J., Martínez, A., Nomelín, C., Saavedra, S., \& Tarquino, A. (2012). Conversacion, conocimiento y convivencia. En R. Pinilla, \& Y. Gutiérrez-Ríos, La Oralidad en contextos diversos. aportes investigativos para su discusión y comprensión. (págs. 430-440). Bogotá: (UD) Universidad Distrital Francisco José de Caldas.

Álzate Ortíz, F. A. (Mayo - Agosto de 2015). Prácticas y formación docente: Un escenario propicio para promover la investigación educativa en Colombia. "Actualidades Investigativas en Educación”, 15(2), 1-17. Recuperado el 15 de marzo de 2019, de https://revistas. ucr.ac.cr/index.php/aie/article/download/18962/19069/

Amber, D., \& Santamarina, M. (2013). La presencia de la oralidad en los planes actuales de grado en Educación Infantil en la comunidad autónoma de Andaluza. En M. Nuñez, J. Rienda, \& M. Santamarina, Oralidad y Educación (págs. 111-122). Granada- España: Monema.
Aramburo, E. (2017). 3er Encuentro Nacional de la Red Iberoamericana de estudios sobre la oralidad. Oralidad y metacognición en el aula. Cali.

Bajtin, M. (1982). Estética de la creación verbal. Siglo XXI.

Beltrán, Y., \& Arango, A. (2012). La oralidad: memoria, proceso organizativo y cotidianidad. ZHct: tradiciones y prácticas dentro del conflicto armado en Colombia. En R. Pinilla, $\&$ Y. Gutiérrez, La oralidad en contextos diversos. Aportes investigativos para su discusión y comprensión (págs. 133-142). Bogotá: (UD) Universidad Distrital Francisco José de Caldas.

Camelo, M. J., Cárdenas, E. J., Torres, M. F., Gutiérrez, Y., Rodríguez, M. E., \& Pinilla, R. (2012). Evaluación de la lengua oral en el aula. Un desafío para los maestros de lengua castellana. En R. Pinilla, \& Y. Gutiérrez, La Oralidad en contextos diversos. Aportes investigativos para su discusión y comprensión (págs. 419-430). Bogotá: (UD) Universidad Distrital Francisco José de Caldas.

Camelo, M., Cárdenas, E., Torres, M., Gutiérrez-Ríos, M. Y., Rodríguez, L., \& Pinilla, V. (2012). Evaluación de la lengua oral en el aula. Un desafío para maestros de lengua castellana. En R. Pinilla, \& Y. Gutiérrez, La oralidad en contextos diversos. Aportes investigativos para su discusión y comprensión. (págs. 419-429). Bogotá: (UD) Universidad Distrital Francisco José de Caldas.

Carvajal, G. (2014). Oralidad y escritura en el contexto escolar: distinciones y articulaciones en las prácticas discursivas. En M. Rodríguez, \& R. Pinilla, Oralidades. Saberes y experiencias de investigación en red (págs. 19-44). Bogotá: (UD) Universidad Distrital Francisco José de Caldas. 
Chaparro, S., \& Camargo, S. (2012). Oralidad con sentido: estrategias orales en el aula. En R. Pinilla , \& Y. Gutiérrez, La Oralidad en contextos diversos. Aportes investigativos para su discusión y comprensión (págs. 441-450). Bogotá: (UD) Universidad Distrital Francisco José de Caldas.

Contreras, I. (2014). Los Albores del lenguaje oral en el devenir de la humanidad. En M. E. Rodríguez, \& R. Pinilla, Oralidades. Saberes $y$ experiencias de investigación en red (págs. 187-198). Bogotá: (UD) Universidad Distrital Francisco José de Caldas.

Delgadillo, I. (2014). Ancestralidad: tradición oral y actualidad. En M. Rodrìguez, \& R. Pinilla, Oralidades. Saberes y experiencias de investigación en red (págs. 233-246). Bogotá: (UD) Universidad Distrital Francisco José de Caldas.

Galeano, J. I. (2012). La oralidad: creencias de maestras en educación inicial. En R. Pinilla, \& Y. Gutiérrez, La Oralidad en contextos diversos. Aportes investigativos para su discusión y comprensión (págs. 503-516). Bogotá: (UD) Universidad Distrital Francisco José de Caldas.

Gil, A. (2017). campo semántico sobre la diversidad en contextos educativos. 3er encuentro Nacional de la red Iberoamericana de estudios sobre la oralidad. Cali.

Gutiérrez-Ríos, M. Y., Lara, F., \& Uribe, R. (04 de Mayo de 2019). Prácticas dialógicas generadoras de pensamiento crítico en la formación de docentes. Colectivo Formación docente y pensamiento Crítico, Red oralidad-Clacso.
Gutiérrez-Ríos, M. Y. (2018 ). Oralidad, diálogo, dialogismo y dialogicidad en la construcción de pensamiento crítico. En J. \&. Llanán Nogueira, Ecos, significados y sentidos. Debates actuales sobre derechos humanos en contextos diversos (págs. 85-96). Rosario, Argentina: Facultad de Derecho de la Universidad Nacional de Rosario.

Gutiérrez-Ríos, M. Y. (2014). Concepciones y prácticas sobre la oralidad en la educación media colombiana. Bogotá: Universidad Distrital Francisco José de Caldas.

Gutiérrez-Ríos, M. Y. (2014). Perspectivas teóricas, tensiones y aperturas de la oralidad contemporánea en Iberoamérica. En S. S. (Comp.), Panorama de los estudios del discurso en Colombia (págs. 183-203). Bogotá. D.C.: Fondo de publicaciones Universidad Distrital Francisco José de Caldas.

Gutiérrez-Ríos, M. Y. (2014). Dialogo y formación docente. En M. Rodríguez, \& R. Pinilla, Oralidades. Saberes y experiencias de investigación en Red (págs. 87-106). Bogotá: (UD) Universidad Distrital Francisco José de Caldas.

Gutiérrez-Ríos, M. Y. (2012). Las concepciones disciplinares y didácticas de los profesores de lengua castellana sobre la lengua oral. En R. Pinilla, \& M. Y. Gutiérrez-Ríos, La Oralidad en contextos diversos. Aportes investigativos para su discusión y comprensión (págs. 559-576). Bogotá: (UD) Universidad Distrital Francisco José de Caldas.

Hamui, S. (2012). La oralidad y performance. En R. Pinilla, \& Y. Gutiérrez, La Oralidad en contextos diversos. Aportes investigativos para su discusión y comprensión. (págs. 43-60). Bogotá: (UD) Universidad Francisco José de Caldas. 
Hernández, M., \& Chávez, J. (2016). Portafolio oral: Una propuesta evaluativa sobre el aprendizaje de la lengua en la educación inicial. En Educación y pedagogía. Aportes de maestros y maestras de Bogotá. (págs. 65-80). Bogotá. Recuperado el 15 de marzo de 2019, de http://www.idep.edu.co/sites/default/ files/libros/Educacion_pedagogia_Aportes_ de_maestros.pdf

Jurado, F. (2014). La oralidad en los curriculos de la educación básica y media en américa latina: avances y retos. En M. Rodríguez, \& R. Pinilla, Oralidades. Saberes y experiencias de investigación en red (págs. 45-56). Bogotá: (UD) Universidad Distrital Francisco José de Caldas.

Kalman, J., \& Rendón, V. (2016). Uso de la hoja de cálculo para analizar datos cualitativos. Magis, Revista Internacional De Investigación En Educación, 9(18), 29-48. https://doi. org/10.11144/Javeriana.m9-18.uhca

León, J. (2015). Hacia una didáctica de la escucha: Más allá de las estrategias.

León, J. (2019). Hermenéutica dialógica: Claves para pensar la escucha en la educación. Folios, $71-81$.

Martínez, A., Moreno, G., \& Vergara, P. (2012). La grabadora Mágica. En R. Pinilla, \& Y. Gutiérrez, La Oralidad en contextos diversos. Aportes investigativos para su discusión y comprensión (págs. 376-384). Bogotá: (UD) Universidad Distrital Francisco José de Caldas.

Martínez, R., Idárraga, M., Ríos, M., \& Ramírez, M. (2016). La familia rural y sus formas de diálogo en la construcción de paz en colombia. Bogotá: Universidad de la Salle.
Méndez, N. (2012). Alfabetización de mujeres y encuentro con su oralidad. En R. Pinilla, \& M. Y. Gutiérrez-Ríos, La oralidad en contextos diversos. aportes investigativos para su discusión y comprensión. (págs. 182-190). Bogotá: (UD) Universidad Distrital Francisco José de Caldas.

Nuñez, M. P. (2012). Espejos y ventanas: dimensiones de la oralidad en el ámbito educativo. En R. Pinilla, \& M. Y. Gutierrez, La oralidad en contextos diversos. Aportes investigativos para su discusión y comprensión (págs. 21-40). Bogotá: Universidad Distrital Francisco José de Caldas.

Nuñez, M., Rienda, J., \& Santamarina, M. (2013). Oralidad y Educación. Granada: Monema.

Obando, L. (2012). Encuentro de voces y recuerdos. En R. Pinilla , \& Y. Gutiérrez, La oralidad en contextos diversos. Aportes investigativos para su discusión y comprensión. (págs. 122-132). Bogotá: (UD) Universidad Distrital Francisco José de Caldas.

Obando, L. (2014). Perspectiva dialógica de la oralidad y de la identidad en las voces infantiles y juveniles. En M. Rodríguez, \& R. Pinilla, Oralidades. Saberes y experiencias de investigación en red (págs. 299-309). Bogotá: (UD) Universidad Distrital Francisco José de Caldas.

Ortega Valencia, P. (2009). La pedagogía Crítica: reflexiones en torno a sus prácticas y sus desafíos. Pedagogía y Saberes, 26-33.

Peña, L. (2012). La Oralidad camino de encuentro y pervivencia sentido en el resguardo indìgena de Yaquivá. En R. Pinilla, \& Y. Gutiérrez, La oralidad en contextos diversos. Aportes investigativos para su discusión y comprensión. (págs. 84-92). Bogotá: (UD) Universidad Distrital Francisco José de Caldas. 
Pinilla, R. (2012). La interacción verbal como marco de referencia para la interpretación de los relatos orales. En R. Pinilla, \& Y. Gutiérrez, La Oralidad en contextos diversos. Aportes investigativos para su discusión y comprensión (págs. 336-346). Bogotá: (UD) Universidad Distrital Francisco José de Caldas.

Pinilla, R. (2014). Las emociones en los relatos orales de niños. En M. E. Rodriguez, \& R. Pinilla, Oralidades. Saberes y experiencias de investigación en red. (págs. 267-282). Bogotá: (UD) Universidad Distrital Francisco José de Caldas.

Pinilla, R., \& Gutiérrez-Ríos, M. Y. (2012). La Oralidad en contextos diversos. Aportes investigativos para su discusión y comprensión. Bogotá: (UD) Universidad Distrital Francisco José de Caldas.

Quitián, S. (2014). Hacia una pedagogía de la lengua oral en el proceso de formación posgradual de maestros en ejercicio. En M. Rodríguez, \& R. Pinilla, Oralidades. Saberes $y$ experiencias de investigación en red (págs. 199-218). Bogotá: (UD) Universidad Distrital Francisco José de Caldas.

Quitían, S. P., \& Gutiérrez-Ríos, M. Y. (2016). Oralidades y cultura. Avances de investigación en Red. Bogotá. Universidad Distrital Francisco José de Caldas.

Rienda, J. (2012). Literatura oral: permanencia y transcendencia en el ámbito educativo. En R. Pinilla, \& Y. Gutiérrez, La oralidad en contextos diversos. Aportes investigativos para su discusión y comprensión. (págs. 201-212). Bogotá: (UD) Universidad Distrital Francisco José de Caldas.

Rincón, C. (2015). Prácticas Pedagógicas que promueven la oralidad en la primera infancia en el marco de una experiencia de investigación y acompañamiento a egresadas.
Rincón, C., Patiño, M., \& Gómez, A. (2017). Aprendizajes discursivos, pedagógicos e investigativos de maestros de Educación Inicial en relación con los usos orales de niños de 2 a 4 años en el marco de la pedagogía de la otredad. 3er Encuentro Nacional de la Red Iberoamericana de estudios sobre la oralidad. Cali.

Rodríguez, M. E., \& Pinilla, R. (2014). Oralidades. Saberes y experiencias de investigación en red. Bogotá: (UD) Universidad Distrital Francisco José de Caldas.

Rodríguez, S. (2012). El trabajo de la oralidad en el aula de transición para construir la voz y las condiciones de ciudadanía. En R. Pinilla, \& M. Y. Gutiérrez-Ríos, La oralidad en contextos diversos. Aportes investigativos para su discusión y comprensión (págs. 408-418). Bogotá: (UD) Universidad Distrital Francisco José de Caldas.

Rojas, G. (2017). Entre la conversación y el diálogo: algunos aspectos para la escucha. Enunciación, 189-201. http://doi. org/10.14483/22486798.11930

Sanagogo, B. (2012). Tradiciones orales: Los Griots Malinkés y el arte oratoria en el Manding. En R. Pinilla, \& M. Y. Gutiérrez-Ríos, La oralidad en contextos diversos. Aportes investigativos para su discusión y comprensión. (págs. 73-83). Bogotá: (UD) Universidad Distrital Francisco José de Caldas.

Santamaría, F., \& Bothert, K. (2012). Relatos de niños: de cómo las vivencias se vuelven palabra. En R. Pinilla, \& M. Y. Gutiérrez, La oralidad en contextos diversos. Aportes investigativos para su discusión y comprensión. (págs. 134-154). Bogotá: (UD) Universidad Distrital Francisco José de Caldas. 
Shiro, M. (2012). El desarrollo de los géneros discursivos en el habla infantil: El caso de la narración. En M. Shiro, P. Charaudeau, \& L. Granato, Los géneros discursivos desde múltiples perspectivas: teorías y análisis. (págs. 249-278). España: Iberoamericana.

Vásquez, F. (2012). Elementos fundamentales para una didáctica de la oralidad. En R. Pinilla, \& M. Y. Gutiérrez-Ríos, La oralidad en contextos diversos. Aportes investigativos para su discusión y comprensión. (págs. 349-360). Bogotá: (UD) Universidad Distrital Francisco José de Caldas.

Vásquez, F. (2014). Oralidad y Educación. Revisiones y propuestas. En M. Rodriguez, \& R. Pinilla, Oralidades. Saberes y experiencias de investigación en red. (págs. 77-86). Bogotá: (UD) Universidad Francisco José de Caldas.

Vásquez, M. (2014). La oralidad en el tercer entorno. oralidad, comunicación audiovisual y comunicación digital. En M. Rodríguez, \& R. Pinilla, Oralidades. saberes y experiencias de investigación en red (págs. 123-134). Bogotá: (UD) Universidad Distrital Francisco José de Caldas.

Villanueva, J. (2012). Formación recibida en el desarrollo de la oralidad en educación infantil y en la enseñanza obligatoria de Andalucia (España): Datos obtenidos a partir de una pequeña muestra. Algunas conclusiones. En R. Pinilla, \& M. Y. Gutiérrez-Ríos, La oralidad en contextos diversos. Aportes investigativos para su discusión y comprensión. (págs. 543-558). Bogotá: (UD) Universidad Distrital Francisco José de Caldas.
Yopasa, M. (2012). Memorias de Barro: Reconstrucción del conocimiento tradicional del entorno natural a través de la historia oral, en Ráquira, Boyacá. En R. Pinilla, \& Y. Gutiérrez, La oralidad en contextos diversos. Aportes investigativos para su discusión y comprensión. (págs. 166-181). Bogotá: (UD) Universidad Distrital Francisco José de Caldas.

Zarate, L., \& Fajardo, L. (2017). La oralidad como herramienta para la construcción de conocimiento. Análisis de una experiencia educativa rural en el primer grado. $3 e r$ Encuentro Nacional de la red Iberoamericana de estudios sobre la oralidad. Cali. 